\title{
RESEARCH ON CONSTRUCTION METHOD OF SPATIO-TEMPORAL DATA MODEL BASED ON BEHAVIOR-EVENT
}

\author{
Jingwen $\mathrm{Li}^{1,2}$, Yuan $\mathrm{Ma}^{2}$,Jianwu Jiang ${ }^{1,2, *}$,Wenda Chen ${ }^{2}$, $\mathrm{Na} \mathrm{Yu}^{2}, \mathrm{Xu} \mathrm{Li}^{2}$ \\ ${ }^{1}$ Guangxi Key Laboratory of Spatial Information and Geomatics, Guilin University of Technology, Guilin 541004, China \\ ${ }^{2}$ Guilin University of Technology, Guilin 541004, China
}

KEY WORDS: Spatial-Temporal Model ,Behavior ,Event , Object-oriented

\begin{abstract}
:
Starting from the object-oriented idea, this paper analyses the existing event-based models and the logical relationship between behavioral cognition and events, and discusses the continuity of behavioral cognition on the time axis from the perspective of temporal and spatial cognition. A geospatial data model based on behavioral-event is proposed. The physical structure and logical structure of the model are mainly designed, and the four-dimensional model of "time, space, attribute and event" is constructed on the axis. The organic combination of the four models can well describe the internal mechanism and rules of geographical objects. The expression of data model based on behavior-event not only elaborates the basic information of geospatial objects, but also records the changes of related events caused by the changes of geographic Entities'behavior, and expresses the relationship between spatial and temporal objects before and after the changes of behavior cognition. This paper also designs an effective method to organize spatiotemporal data, so as to realize the effective management and analysis of spatio-temporal data and meet the requirements of storage, processing and mining of large spatio-temporal data.
\end{abstract}

\section{INTRODUCTION}

\subsection{General Instructions}

With the advent of the era of big data, the new generation of GIS must have the ability to effectively express, manage and analyze large geo-spatial data. Spatio-temporal model is the basis and key to solve the above problems.Spatio-temporal model can effectively organize and manage the attributes, spatial and temporal semantics of Temporal Geographic data ${ }^{[1]}$. It is the basis of temporal GIS (TGIS) to reasonably and effectively express the temporal and spatial changes of entities in the real world and organize their spatial and temporal relations. At present, the standardized Spatio-temporal data model is still in the exploratory stage ${ }^{[2]}$. Object-oriented technology is the most effective way to support complex object modeling in space. A geographic entity, no matter how complex, can always be modeled as an object.

Many scholars at home and abroad have done in-depth research on TGIS and proposed a large number of spatiotemporal data models. Peu-quet and Duan proposed an event-oriented spatiotemporal model. For the first time, events were used to describe the time-varying characteristics of objects, and event-based retrospective method was designed to realize the dynamic changes in time segments ${ }^{[3]}$. Xiaolu Chai proposed dividing the object involved in the event-based model into three domains to ensure the continuity of object change ${ }^{[4]}$. Zhihong $\mathrm{Xu}$ and Fuling Bian further divide the objects involved in the eventbased model into five domains, each of which is related by object markers. ${ }^{[5]}$ These models are constructed from the perspective of computer, based on the theory of event cognition, and they can't well express the causal relationship of geographical phenomena.

With the continuous development of TGIS, the existing Spatiotemporal model has some shortcomings in the dynamic change of geographical entities and the expression of their correlation, which is difficult to meet the needs. From the point of view of behavior cognition and event semantics of geographic objects, this paper describes the causality before and after the change of geographic space-time objects by constructing a Spatiotemporal data model based on behavior-event. It predicts the future of geographic phenomena according to the reasons and trends of change, and organizes time with object-oriented thinking. Spatial, attribute and event information, and an effective method for spatiotemporal data organization is designed to satisfy the operation of spatiotemporal data in storage and query.

\section{BASIC RELATIONS OF SPATIO-TEMPORAL MODEL}

\subsection{Event description}

Events are the source of all changes in the world. Events lead to the constant change of entity state, which covers all aspects of entity change, including the change of object state, the change of spatial location, the change of object attributes and the evolution of objects.

Because of the one-dimensional fluidity of time, spatial semantics and thematic semantics describe things on the basis of temporal semantics. The more detailed the semantic description, the more comprehensive the semantics and attributes of geographic events are. Once things change in space and attributes, they may produce one or more events, so the degree of event description plays an indispensable role in the process of spatial and temporal evolution analysis. Every geographic event occupies the space domain or the time domain under a certain time, and the time and place of its existence and disappearance are uncertain ${ }^{[7]}$. The expression of event semantics can not only describe the temporal, spatial, temporal, thematic attributes of geospatial objects and the relationship

* Jianwu Jiang - E-mail: fengbuxi@glut.edu.cn 
between temporal and spatial changes, but also record the process of the origin of the change of things, so as to realize the full expression of the process of geospatial and temporal changes.

The relationship between events and other tuples is shown in Figure 1:

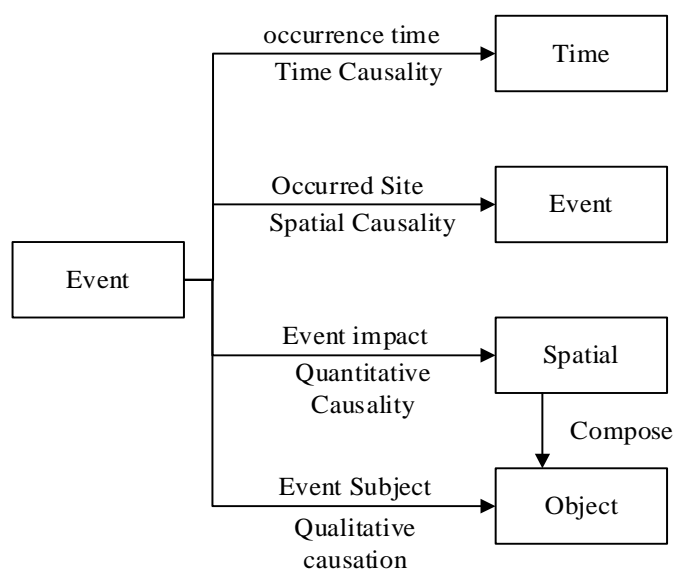

Figure 1 Event Diagram

\subsection{The Relation between Events and Behaviors}

Events and behaviors are a pair of important concepts in spatiotemporal databases. Behavior is the description of things changing, and events are the cause of things changing ${ }^{[8]}$. The change of things in their life cycle is driven by events, which is a state and a behavior from trigger to termination. It has continuity on the time axis, but its spatial characteristics are discrete sets, which are composed of spatial attributes of multiple different times. Assuming that the change of each spatial attribute represents the occurrence of an event, the behavior can be expressed as a set of events generated by a geographical entity in different spatial attribute characteristics.

Assuming that behavior cognition is $\mathrm{B}$, according to the hierarchical relationship between behavioral cognition and event logic, it can be expressed as follows:

$$
\mathrm{B}=\{(\mathrm{E}, \mathrm{T})\}=\left\{\left(e_{1}, t_{1}\right),\left(e_{2}, t_{2}\right), \ldots,\left(e_{n}, t_{n}\right)\right\}
$$
In equation $(1),\left(e_{n}, t_{n}\right)$ denotes that the change of events is the independent variable and the behavior on time $t_{n}$ is the dependent variable. $\left(t_{1}, t_{n}\right)$ can be a discrete time point or a continuous time period on time set $\mathrm{T}$. With the change of a behavioral cognition, the corresponding events also change. The relationship between them belongs to $1: n$. As shown in the figure2.

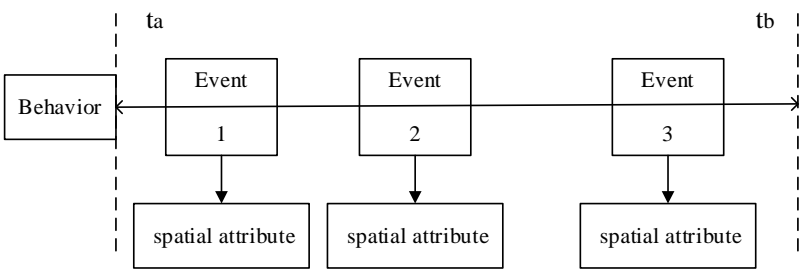

Figure 2. The relationship between behavior and events

\subsection{Spatio-temporal Model Based on Event}

Spatio-temporal Model Based on Event is a model proposed by Peuquet in 1995 for raster data. The model fully stores the grid base map. Consider the changes made at each moment as an event, and record the time of the event and the changes made in detail. Record the location of the change and the value of the change. As shown in Figure 3, assuming that a state of the map lasts for $\left[T_{n}, T_{n+1}\right]$, that is, events occurring at $T_{n+1}$ time change the state of the map and put the map in a new state. The map with $T_{n+1}$ time is equal to the change of $T_{n}$ time map and $T_{n+1}$ event. Therefore, if we want to get the map with $\mathrm{F}$ time, we need to get the map with $T_{n}$ time first. So when loading the latest map data, you need to read the base map data and review the changes made by previous events. With the passage of time, the number of event records increases gradually. Loading current map data will become more and more complex, seriously affecting the efficiency of the system ${ }^{[9]}$.

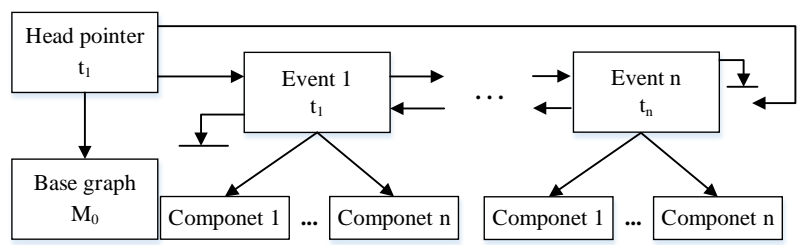

Figure 3 Event-based spatiotemporal data model

\section{CONSTRUCTION OF B-E GEOGRAPHIC CHARACTERISTIC MODEL}

\subsection{Model introduction}

The Geographic Information Model based on Behavior-Event includes four aspects: attribute feature, time feature, spatial feature and event feature. Time dimension is the basis of the other three dimensions. Time dimension runs through the three dimensions of space, attributes and events. It is the bottom of the three dimensions. That is to say, each dimension has time attributes when it is expressed, all within the three scope of birth, existence and extinction of time dimension. Combining attribute dimension, time dimension, spatial dimension and event semantics, the data cube composed of event and attribute dimension and spatial-temporal data cube based on time stamp are stored.

Spatio-temporal data model based on behavior-event not only expounds the basic information of geographic spatial-temporal objects, but also changes the related events by recording the changes of geographic Entities'behavior, expressing the relationship between spatial-temporal objects before and after the change of behavior cognition, and also expressing the time before and after the change of objects. The model is shown in the figure4. 


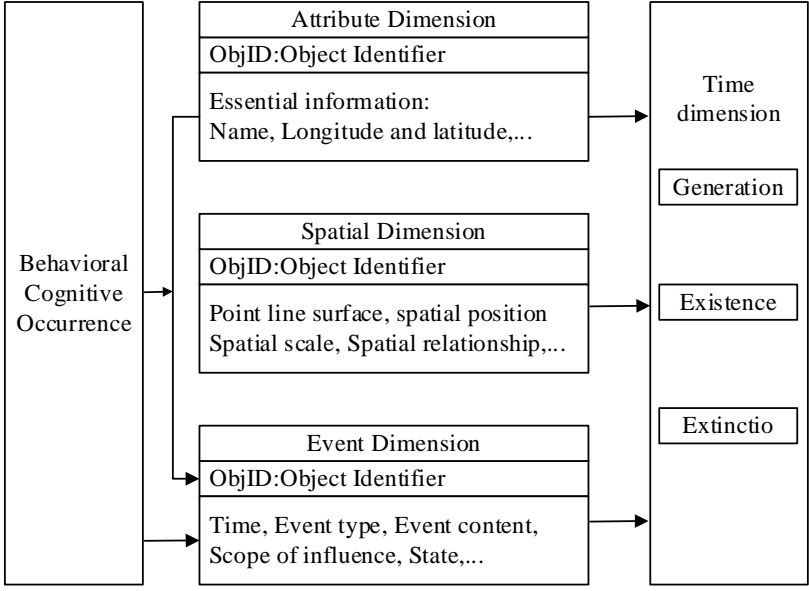

Figure 4. Behavior-event model

\subsection{Conceptual Model}

In this paper, object-oriented method is used to formalize the geographic reality according to the spatial-temporal semantic relationship between concepts. According to Gruber's definition of ontology, this model combines temporal semantics, spatial semantics and thematic semantics of the model and abstracts them into a six-tuple to describe the temporal and spatial changes of geographical entities.

$$
<\text { Object, }\left\{O I D, S(\mathrm{t}), \mathrm{A}(\mathrm{t}), \mathrm{T}\left(T_{s}, T_{e}, T_{d}\right), \text { Opera }\right\}>
$$

OID is the object identification code of geographic object $\mathrm{O}$, which has uniqueness in the whole space-time and remains unchanged all the time.

$\mathrm{S}(\mathrm{t})$ denotes the set of spatial characteristics of space-time objects varying with time in a specific space coordinate system, $\mathrm{S}(\mathrm{t})=\left\{\left(\vec{q}_{1}, t_{1}\right),\left(\vec{q}_{2}, t_{2}\right), \ldots,\left(\vec{q}_{n}, t_{n}\right)\right\}$, where $\vec{q}_{i}=\left\{q^{1}, q^{2}, \ldots q^{n}\right\}$ denotes the set of spatial data types of space-time objects and $q^{k}=\left\{\left(x_{1}, y_{1}, z_{1}\right),\left(x_{2}, y_{2}, z_{2}\right), \ldots,\left(x_{k}, y_{k}, z_{k}\right)\right\}$ denotes the sequence of discrete point coordinates.

$\mathrm{A}(\mathrm{t})=\left\{\left(p_{1}^{1}, p_{2}^{1}, \ldots, p_{m}^{1}, t_{1}\right),\left(p_{1}^{2}, p_{2}^{2}, \ldots, p_{m}^{2}, t_{2}\right), \ldots\right\} \quad$ represents the set of attributes of object $\mathrm{O}$ over time. $p_{j}^{i}$ represents the state of the $\mathrm{j}$-th attribute property of space-time object $\mathrm{O}$ at time $t_{i}$, which $\mathrm{j} \in[1, \mathrm{~m}], \mathrm{i} \in[1, \mathrm{n}]$

$\mathrm{T}\left(T_{s}, T_{e}, T_{d}\right)$ denotes the temporal nature of the change of the state of the spatiotemporal object, where $T_{s}$ denotes the initial time of the change of the state of the object, $T_{e}$ denotes the end time of the state of the object, and $T_{d}$ denotes the time of the database.

Opera represents all kinds of behavior operations on space-time objects, that is, the properties of objects and the operation of time and space.

\subsection{Logical Model}

The logical data model is based on the conceptual data model to determine the detailed logical structure of each component of the conceptual model. As shown in figure 5, each space-time object contains information about space, time, attributes, and event characteristics. Four feature information can be abstracted into spatial class, temporal class, attribute class and event class.
Where Spatial Class is an abstract expression of the position information and geometric state of a geographical entity, which is composed of Spatial Coordinate Class and Geo-relationship Class. Temporal Class consists of Temporal Coordinate Class and Time Class. Attribute Class consists of Dynamic Attribute Class and Static Attribute Class. Event Class consists of Event Process Class and Event Element Class. Classes also encapsulate object identification and corresponding behavior operations, specifically the space, attributes, time, events and their joint operations. These four object classes are associated by object identifier OID.

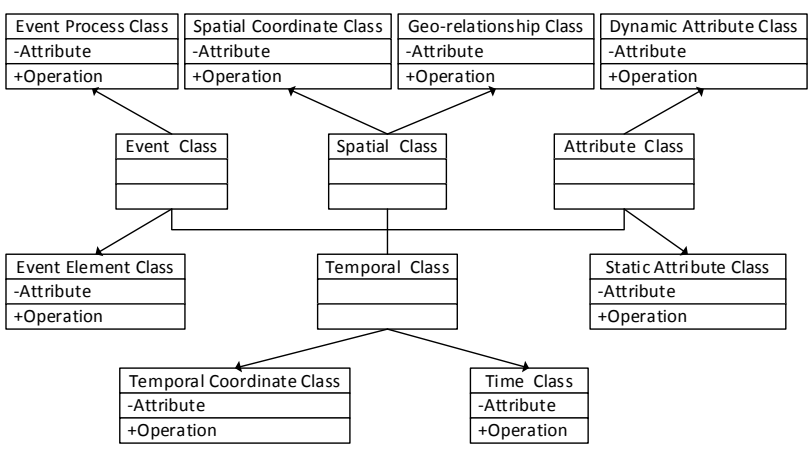

Figure 5. Logical Structure Diagram of Multidimensional Feature Model

\subsection{Data organization}

In daily spatio-temporal data management, most of the data operations are directed at the current data, and the direct operation of historical data generally occurs in the query of historical data ${ }^{[11]}$. If the current data and historical data are stored in the same database, it will lead to duplication of data storage and inefficiency of the system. Therefore, it is necessary to optimize the organization of data.

In this paper, an effective method is designed for the organization of spatial-temporal data: according to the different properties of the data, the geographical spatial-temporal database is divided into the present database, the event database, the historical database and the behavior database. The present database stores the latest status data; the event inventory stores the relevant information of each event that causes the geographical entity to change; the historical inventory stores the historical data of the updated geographical object before the update, including the event identification number that causes the data update, through which the historical behavior of the data update can be obtained. The behavior database preserves the historical behavior of each time period of temporal and spatial data.

When an event occurs, the event and the corresponding behavior identifier of the event are stored in the event library. At the same time, all the changed geographical entities and the event identifier are stored in the history library. When querying, firstly, the event and its behavior identifier are queried in the event library. According to the event identifier, the changed geographic object is extracted from the history library, and the unchanged geographic object identified by the behavior identifier is combined and calculated to restore the state of the geographic object at that time. 


\section{CONCLUSION}

In this paper, from the angle of the time-space data information on the time axis, the logical relation between the event and time, the relationship between the event and the behavior, the timespace data model based on the behavior and the time, and the organization and storage of the space-time data are optimized. Through the analysis of the time-varying sequence and the deep-level cause of the change of the characteristic of the thing, the description and the expression method of the time-space data model based on the behavior-based event are discussed, and the method can store the change data in the historical event in time, At the same time, the series of events and processes that cause the change of the state of the thing can not only be solved, but also the whole problem of the historical data and the current data after the event change can be solved, and the query and analysis based on the time-space change semantics can be realized. Behavior-event based spatiotemporal data model improves the limitations of traditional event model in historical retrospect and future prediction, but it also has some shortcomings. It does not fully take into account the combination relationship between other semantics, can't express the non-temporal spatial topological relationship, and has not done relevant research on its change process. These problems will be further considered in future studies.

\section{REFERENCES}

[1] Guanfu Song, Ershun Zhong, Zhifeng $\mathrm{Wu}$, Shaojun Li,Wenwen Cai and Wang Shaohua. Four key technologies of the new generation of basic GIS software [J]. Surveying and mapping geographic information, 2019, 44 (01): 1-8.

[2] Shu Hong, Chen Jun, Du Daosheng, Zhou Yongqian. Object-oriented spatiotemporal data model [J]. Journal of Wuhan University of Surveying and Mapping Science and Technology, 1997 (03): 43-47.

[3] Peuquet D J,Duan N.An Event-Based Spatio-Temporal Data Model (ESTDM) for Temporal Analysis of Geographical Data[J].International Journal of Geographical Information System,1995,9(1):7 24.

[4] Xiaolu Chai, Cao Jing, Bole Shi . A Hierarchical Model of Spatio-temporal Information Storage Management [C]//16th National Conference on Database. 1999.

[5] Xu Zhihong, Shentu Harbour, Bianfuling. Spatiotemporal Modeling of Cadastral Management Information System Based on Event Semantics [J]. Geographic and Geographic Information Science, 2004 (04): 6-10.

[6] Sun Yan. Research and application of event-based objectoriented spatiotemporal data model [D]. Dalian: Dalian University of Technology, 2007.

[7] Xinbao Chen, Li S, Li Li ,et al. Spatiotemporal Data Model Based on Object-Event-Process and Its Application [J].Geographic and Geographic Information Science, 2013, 29 (3): $14-20$.

[8] Zhang Yun, Xuezhi Feng, Jiangfeng She. Study on GeoSpatial-Temporal Data Model Based on Event-State [J]. Mapping and Spatial Geographic Information, 2009, 32 (6): 5-8.
[9] Guangfa Lin, Xuezhi Feng ,Wang Lei, et al. Objectoriented spatio-temporal data model with event as its core [J]. Journal of Surveying and Mapping, 2002, 31 (1): 71-76.

[10] Gruber T A ,Translation Approach to Portable Ontology Specifications . Knowledge Acquisition,1995

[11] Zhangcai Yin, Li Lin. Spatio-temporal data model in GIS [J]. Surveying and Mapping Science, 2005, 30 (3): 12-14. 